CARDIOLOGY

\title{
Rationale and Design of the 'MITOCARE' Study: A Phase II, Multicenter, Randomized, Double-Blind, Placebo-Controlled Study to Assess the Safety and Efficacy of TRO40303 for the Reduction of Reperfusion Injury in Patients Undergoing Percutaneous Coronary Intervention for Acute Myocardial Infarction
}

\author{
MITOCARE Study Group ${ }^{1}$
}

\section{Key Words \\ Acute myocardial infarction - EU-FP7 grant $\cdot$ Percutaneous coronary intervention $\cdot$ Reperfusion injury $\cdot$ TRO40303}

\begin{abstract}
Treatment of acute ST-elevation myocardial infarction (STEMI) by reperfusion using percutaneous coronary intervention $(\mathrm{PCl})$ or thrombolysis has provided clinical benefits; however, it also induces considerable cell death. This process is called reperfusion injury. The continuing high rates of mortality and heart failure after acute myocardial infarction (AMI) emphasize the need for improved strategies to limit reperfusion injury and improve clinical outcomes. The objective of this study is to assess safety and efficacy of TRO40303 in limiting reperfusion injury in patients treated for STEMI. TRO40303 targets the mitochondrial permeability transition pore, a promising target for the prevention of reperfusion injury. This multicenter, double-blind study will randomize patients with STEMI to TRO40303 or placebo administered just before balloon inflation or thromboaspiration during $\mathrm{PCl}$. The primary outcome measure will be reduc-
\end{abstract}

1 See Appendix for the full list of MITOCARE Investigators. tion in infarct size (assessed as plasma creatine kinase and troponin I area under the curve over 3 days). The main secondary endpoint will be infarct size normalized to the myocardium at risk (expressed by the myocardial salvage index assessed by cardiac magnetic resonance). The study is being financed under an EU-FP7 grant and conducted under the auspices of the MITOCARE research consortium, which includes experts from clinical and basic research centers, as well as commercial enterprises, throughout Europe. Results from this study will contribute to a better understanding of the complex pathophysiology underlying myocardial injury after STEMI. The present paper describes the rationale, design and the methods of the trial.

Copyright $\odot 2012$ S. Karger AG, Basel

\section{Introduction}

Current treatment of acute ST-elevation myocardial infarction (STEMI) by reperfusion using percutaneous coronary intervention (PCI) or thrombolysis provides tremendous clinical benefits by reducing infarct size and mortality following cardiac ischemia. However, reperfusion also induces considerable cell death. This process is called reperfusion injury and is believed to ac-

Prof. Dan Atar, MD

Department of Cardiology, Oslo University Hospital Ullevaal

University of Oslo, Institute for Clinical Sciences

NO-0407 Oslo (Norway)

E-Maildan.atar@online.no 
count for about $50 \%$ of the remaining infarction [1]. The continuing high rates of mortality $(\sim 10 \%)$ and heart failure after acute myocardial infarction (AMI; 18\%) [2] emphasize the need for improved strategies to limit reperfusion injury in order to further improve clinical outcomes.

Several strategies for the reduction in reperfusion injury, such as post-conditioning [3], cyclosporine A [4] and FX06 [5], have been studied in phase II trials in patients undergoing PCI. Although these therapies have demonstrated the potential to reduce infarct size measured by some cardiac parameters, results were not consistent for all biomarkers or cardiac magnetic resonance (CMR) measurements.

In the clinical trial with cyclosporine $\mathrm{A}$, a significant $40 \%$ reduction in the plasma creatine kinase (CK) area under the curve (AUC) from 0 to $48 \mathrm{~h}$ was observed together with a $20 \%$ reduction in infarct size assessed by MRI [4]. However, the effect on plasma troponin I, which is considered a more specific marker for myocardial damage than CK, was not statistically significant. Similarly, FX06 resulted in a significant $58 \%$ reduction in the necrotic core zone assessed by MRI, but no significant change in late gadolinium enhancement (LGE), mean troponin I plasma levels or the CK myocardial band activity [5]. The efficacy of post-conditioning remains controversial despite positive results in early studies [3, 6-10]. Subsequent trials did not report a reduction in infarct size, but rather found that post-conditioning might have potential harmful effects $[11,12]$.

TRO40303 (3,5-seco-4-nor-cholestan-5-one oxime-3$\mathrm{ol}$ ), a mitochondrial permeability transition pore (mPTP) modulator, was identified in a cell-based assay designed to identify molecules that maintain survival of trophic factor-deprived motor neurons [13]. It binds specifically to the mitochondrial translocator protein at the cholesterol site and has the potential to decrease mitochondrialmediated cell death.

TRO40303 has been shown to reduce infarct size in preclinical animal models [14]. It specifically targets reperfusion-related events, delaying $\mathrm{mPTP}$ opening triggered by hypoxia reoxygenation in cardiomyocytes in vitro and reducing the release of apoptosis-inducing factor into the cytosol after in vivo ischemia reperfusion [14]. Since TRO40303 targets specific reperfusion events, it has the potential to prevent or limit myocardial reperfusion injury, evolution to heart failure and the subsequent need for regenerative therapies.

The MITOCARE consortium, which began in January 2011, is a group of experts from 9 clinical centers, 3 basic research centers and 4 small and medium-sized enterprises, for a total of 16 teams throughout Europe specializing in clinical and basic research, biomarkers, imaging and informatics. This EU-FP7 (EU-7th Framework Program)-funded consortium focuses its expertise on the problem of cardiac ischemia reperfusion injury.

\section{Study Rationale}

This trial seeks to evaluate the efficacy and safety of a new drug, TRO40303, in patients presenting early with a STEMI, the period when reperfusion therapy has the greatest benefit. TRO40303 targets the MPTP, a highly promising target for the prevention of reperfusion injury $[15,16]$. The primary outcome measure will be reduction in infarct size, which is known to be an important surrogate endpoint for morbidity and mortality following AMI $[17,18]$.

In the present phase II trial, TRO40303 or placebo will be given, as an adjunct to current standard cardiac care, to patients undergoing primary PCI to treat STEMI. Results from this study will contribute to a better understanding of the complex pathophysiology underlying myocardial injury after STEMI.

\section{Study Objectives}

The objective of the study is to assess the safety and efficacy of TRO40303, which is administered just before balloon inflation or thromboaspiration during PCI for the limitation of infarct size in patients treated for STEMI. Comparative efficacy between TRO40303- and placebo-treated patients will be assessed using cardiac biomarkers and cardiac imaging. The co-primary measures of efficacy will be infarct size expressed as AUC for CK and AUC for troponin I (used for sample size calculation) over 3 days.

The main secondary outcome measure will be infarct size normalized to the myocardium at risk, expressed as 'myocardial salvage index', evaluated by CMR. Additional secondary outcome measures to be compared between TRO40303- and placebo-treated groups include: left ventricular function after PCI measured by CMR (days 3-5) and echocardiography (days 3-5 and 30); ST-segment decrease after PCI (ECG before PCI compared to an ECG performed $1 \mathrm{~h}$ after stenting); magnitude of microvascular obstruction measured by CMR and transmural extension of the infarct measured by CMR. 
Table 1. Additional biomarker measures

\begin{tabular}{ll}
\hline Cardiac function/vascular biomarkers & $\begin{array}{l}\text { troponin } \mathrm{T}, \mathrm{CK}-\mathrm{MB} \text { : creatine phosphokinase-MB, N-terminal prohormone of brain } \\
\text { natriuretic peptide, pro-adrenomedullin }\end{array}$ \\
\hline Renal function biomarkers & cystatin C, microalbuminuria \\
\hline Inflammation biomarkers & C-reactive protein, interleukins $1 \mathrm{~b}, 6$ and 10, immunoglobulins, tumor necrosis factor- $\alpha$ \\
\hline MOA-related biomarkers & cytochrome $\mathrm{C}$, oxidized LDL, myeloperoxidase \\
\hline Diabetes biomarker & hemoglobin $\mathrm{A}_{1 \mathrm{c}}$ \\
\hline Platelet activation & thromboxane in urine \\
\hline Exploratory biomarkers & at least three exploratory biomarkers \\
\hline
\end{tabular}

$\mathrm{LDL}=$ Low-density lipoprotein $\mathrm{MB}=$ myocardial band.

A subgroup analysis of infarct size (evaluated by cardiac biomarkers and cardiac imaging) will be conducted according to the culprit vessel. In addition, patients will be classified according to Killip class and GRACE score.

Safety will be assessed at baseline, and 3 and 30 days after PCI. Measures include physical examination (clinical symptoms and weight), vital signs (blood pressure and heart rate), serum creatinine, triglycerides, urea, blood glucose, electrolytes, serum bilirubin, $\gamma$-glutamyltransferase, alkaline phosphatase, amino transferase, alanine amino transferase and high/low density lipoprotein cholesterol, as well as hematology and blood coagulation tests. Cumulative incidence of major adverse events occurring during the first $48 \mathrm{~h}$ after reperfusion, including death, heart failure, AMI, stroke, recurrent ischemia, the need for repeat revascularization, renal, hepatic or vascular complications, and bleeding, will be assessed. Cardiac and non-cardiac events occurring within the initial 30 days will be recorded.

Additional biomarker measures are listed in table 1. These markers will be used to assess factors for potential confounding influence or prognostic value for infarct size, future progression to heart failure, risk stratification, mechanism of cardioprotection and variability of response. Biomarker assays are centralized. In addition, a biobank of patients' plasma, serum and RNA will be established for further investigations.

\section{Study Design}

MITOCARE is a prospective, multicenter, randomized, double-blind, placebo-controlled, phase IIa, proofof-concept study in patients with large STEMI undergo- ing primary $\mathrm{PCI}$, where TRO40303 or placebo will be administered as a single intravenous bolus prior to the reperfusion by angioplasty.

\section{Patient Population}

The study will be conducted in 9 study centers across 4 European countries. A planned total of 180 patients will be assigned 1:1 to receive either TRO40303 or placebo. The study population includes patients $>18$ years of age with a first-time AMI. Patient inclusion and exclusion criteria are listed in table 2.

\section{Study Interventions}

Eligible patients will undergo primary PCI and receive concomitant medications according to the current standard of care. After coronary angiography, but just before balloon inflation or thromboaspiration, patients who meet the enrolment criteria will be randomized to receive either placebo or TRO40303 (fig. 1). Randomization is ensured by taking the treatment units in ascending and consecutive order in each infarction stratum (anterior/ posterior as determined on ECG).

Patients will receive an intravenous slow-bolus $(35 \mathrm{ml} /$ $\mathrm{min}$ ) injection of TRO40303, $6 \mathrm{mg} / \mathrm{kg}$, or an equivalent volume of placebo, a maximum of $15 \mathrm{~min}$ (ideally $<5$ $\mathrm{min}$ ) before balloon inflation and stenting. Patients will subsequently undergo in- or outpatient CMR and echocardiography between days 3 (ideally) and 5. Follow-up safety assessment and echocardiography will be performed 30 days after PCI. Patients will remain in hospital as long as medically indicated.

Since this clinical trial is being conducted within the context of an acute medical emergency, traditional case report forms (CRF) will be replaced with a new electronic version (eCRF) available on portable devices (iPad ${ }^{\mathrm{TM}}$ ) 
Fig. 1. Study design.

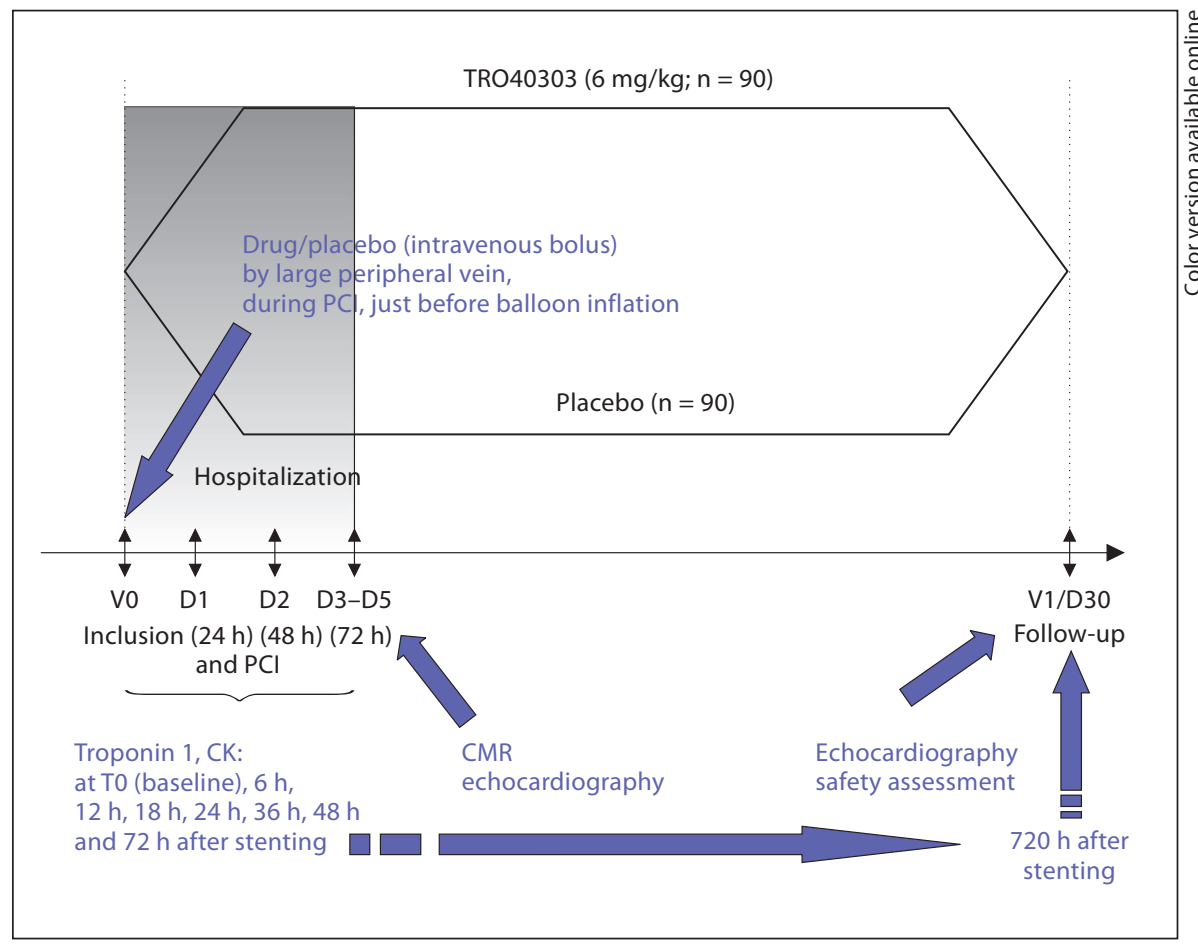

Table 2. Inclusion and exclusion criteria

\section{Inclusion criteria}

Age $>18$ years old

Male or female with non-child-bearing potential ${ }^{\mathrm{a}}$

First AMI defined as:

Nitrate-resistant chest pain $\geq 30 \mathrm{~min}$

New ST-elevation at J-point in two contiguous leads with cutoff points: $\geq 0.2 \mathrm{mV}$ in men or $\geq 0.15 \mathrm{mV}$ in women in leads $\mathrm{V} 2$ - V3 and/or $\geq 0.1 \mathrm{mV}$ in other leads

Presenting within $6 \mathrm{~h}$ of onset of chest pain

Clinical decision to treat with PCI

Occlusion of culprit artery with TIMI flow 0-1 prior to PCI

Occlusion affecting the following coronary arteries: left anterior descending artery, or dominant or balanced right coronary artery, or dominant or balanced left circumflex artery

Signed informed consent to participate in the trial before any study-related procedure or given oral consent in France and in Norway (patient should

sign the informed consent form within $12 \mathrm{~h}$ after the procedure)

\section{Exclusion criteria}

Cardiac arrest, ventricular fibrillation, cardiogenic shock, stent thrombosis, previous AMI, angina within $48 \mathrm{~h}$ before infarction, previous CABG, intravenous fibrinolytic therapy within $72 \mathrm{~h}$ prior to PCI

Atrial fibrillation

Pacemaker

Concurrent inflammatory, infectious or malignant disease

Biliary obstruction or hepatic insufficiency

History or presence of egg allergy

Claustrophobia at MR-scan

a Postmenopausal, ovariectomized or hysterectomized patients. Menopause defined as age $>60$ years, or between 45 and 60 years being amenorrheic for $\geq 2$ years. TIMI = Thrombolysis in myocardial infarction; CABG = coronary artery bypass graft. 
and adapted for an emergency setting. This will allow for greater ease of data collection and reduce the possibility of errors in study monitoring.

\section{Cardiac MRI}

Cardiac imaging has been shown to allow for assessment of infarct size and heart function in acute ischemic heart disease [19]. CMR using LGE is currently considered the clinical method of choice for detailed depiction of myocardial infarction [20,21]. Clinically, imaging techniques are not systematically applied, primarily due to cost limitations; however, they are very useful in establishing the efficacy of new cardioprotective agents.

Infarct size in man is influenced by the duration of ischemia and by the size of the myocardium at risk [22], which is dependent on the site of the coronary occlusion. CMR has been shown to enable quantification of the myocardium at risk using T2-weighted CMR [23]. This technique has also been clinically validated against myocardial perfusion single-photon emission computed tomography and shown to depict the myocardium at risk up to 1 week after the acute coronary event [24]. Furthermore, contrast-enhanced steady-state free precession has recently been shown to also enable depiction of the myocardium at risk 1 week after the acute event $[25,26]$. The main secondary endpoint in this study is the infarct size normalized to the myocardium at risk, expressed as the myocardial salvage index. Use of this parameter measures the efficacy of treatment, but also provides data on patient variability and allows comparisons with preclinical data where infarct size is usually evaluated as the percentage of the area at risk.

Infarct size will be assessed from the LGE CMR images using a previously validated quantification method [27]. To avoid overestimation of infarct size by LGE CMR in the first days after the acute event $[28,29]$, the CMR will be performed 3-5 days later. The myocardium at risk will be assessed either by T2-weighted imaging [24] or contrast-enhanced steady-state free precession imaging [25]. All image analysis will be performed by ImaCor AB (Lund, Sweden). The CMR imaging will be performed on whole-body MR scanners with cardiac applications used for standard clinical CMR.

\section{Statistical Considerations}

The sample size calculation was based on a standardized difference of 0.47 , estimated from data in the study by Piot et al. [4]. Assuming a reduction of $35 \%$ in troponin
I AUC, for a statistical power of $85 \%$ and a probability of type I error of 0.05 using a two-sided test, the sample size was computed to be 166 patients (83 per group), which has been rounded to 180 .

Between-group comparisons of AUC for serum troponin I and CK release will be performed using O'Brien's method for multiple-endpoint testing. Comparisons of CMR data will be performed using an analysis of covariance mixed model. Centers and patients will be considered as random effects and treatment as a fixed effect. Time to PCI, area at risk and culprit artery will be used as covariates for these analyses. All analyses will be performed on the intent-to-treat and per-protocol populations.

For the safety analyses, a comparison of the incidence of cumulative adverse clinical events between treatment groups will be performed using Fisher's exact tests.

\section{Trial Organization}

\section{Steering Committee}

The clinical research steering committee is responsible for supervising the trial and shall provide advice to the project coordinator (Trophos) pertaining to key decisions for the good management of the clinical trial. This committee will also bear overall responsibility for the design, conduct and supervision of the study. It is responsible for reviewing the progress of the study at regular intervals to guarantee safety and trial integrity. The committee will also be responsible for providing clinical guidance on how to interpret the study protocol, study implementation and elucidation of results.

\section{Data Management and Statistical Analysis}

All data management- and statistical analysis-related tasks will be conducted by the Clinical Research Unit, Lariboisière Hospital, Paris, France, with the resultant outcome and safety data being continually provided to the independent drug safety monitoring firm. A new eCRF will be created by Mobile Health for use on the portable iPad device.

CMR images will be transferred from the study centers to a central image database using a new software platform developed by the consortium. Images are automatically anonymized and scanned for inconsistencies before being electronically transferred. As soon as images are transferred, a quality assurance manager on call is notified via text message. Images can be reviewed on any web-enabled mobile device, allowing for direct and fast 
feedback to sites regarding image quality and potential deviations from imaging protocol, thus potentially minimizing the risk of missing patient data due to inadequate imaging.

\section{Data Monitoring Committee}

Two clinicians and a statistician will take part in a data monitoring committee, tasked with ensuring patient safety during the trial. The committee will regularly be provided with safety and efficacy data in a semi-blind way, as well as details of all serious adverse events.

\section{Clinical Event Committee}

The clinical events committee is made up of interventional and non-interventional cardiologists who are not participants in the study. It will be responsible for the adjudication of cardiovascular events based on review of CRF data, serious adverse event reports and source documents (hospital discharge summary, ECG and other supporting documents).

\section{Safety and Ethics Monitoring Committee}

The safety and ethics monitoring committee is in charge of ethics and personal data protection in the clinical trial. It is composed of a data protection specialist and an ethics specialist.

\section{Clinical Implications}

Previous data show that TRO40303 targets a key event that occurs at the time of reperfusion, namely $\mathrm{mPTP}$ opening, making this agent a promising option in reducing reperfusion injury. The MITOCARE project aims to provide in vivo validation of $\mathrm{MPTP}$ as a relevant target for the prevention of myocardial ischemia reperfusion injury and evidence that these mitochondrial processes are modifiable in humans. Effective reduction of reperfusion injury using TRO40303 should decrease the need for regenerative treatments, reduce the development of heart failure and, importantly, reduce mortality.

The MITOCARE project will help increase the understanding of the pathology and evolution of AMI by identifying and extensively analyzing patient-related confounding factors. Identification of new biomarkers will enhance the subsequent development of better tools for the diagnosis and follow-up of AMI. in preclinical models of AMI in order to better refine the understanding of these models, including their utility and

\section{Appendix}

\section{MITOCARE Investigators}

Dan Atar, Department of Cardiology, Oslo University Hospital Ullevål, University of Oslo, Oslo, Norway; Jean-Louis Abitbol, Trophos SA, Luminy Biotech Enterprises, Marseille, France; Hakan Arheden, Department of Clinical Physiology, Lund University, Skåne University Hospital, Lund, Sweden; Alain Berdeaux, Université Paris Est Val de Marne, Créteil, France; Jean-Louis Bonnet, Assistance Publique Hôpitaux de Marseille, Hôpital La Timone, Marseille, France; Marcus Carlsson, Department of Clinical Physiology, Lund University, Skåne University Hospital, Lund, Sweden; Peter Clemmensen, Department of Cardiology, Rigshospitalet, Copenhagen University Hospital, Copenhagen, Denmark; Valérie Cuvier, Trophos SA, Luminy Biotech Enterprises, Marseille, France; Nicolas Danchin, Assistance Publique Hôpitaux de Paris, Hôpital Européen Georges Pompidou, Paris, France; Jean-Luc Dubois-Randé, Assistance Publique Hôpitaux de Paris, Hôpital Henri Mondor, Créteil, France; Henrik Engblom, Department of Clinical Physiology, Lund University, Skåne University Hospital, Lund, Sweden; David Erlinge, University of Lund, Lund, Sweden; Hüseyin Firat, Firalis SAS, Huningue, France; Svend Eggert Jensen, Region Nordjylland Aalborg, Denmark; Sigrun Halvorsen, Department of Cardiology, Oslo University Hospital, Oslo, Norway; Henrik Steen Hansen, Department of Cardiology B, Odense University Hospital, Odense, Denmark; Einar Heiberg, Department of Clinical Physiology, Lund University, Skåne University Hospital, Lund, Sweden; Alf-Inge Larsen, Division of Cardiology, Stavanger University Hospital, Stavanger, Norway; Philippe Le Corvoisier, Assistance Publique Hôpitaux de Paris, Hôpital Henri Mondor, Centre d'Investigation Clinique, Créteil, France; Pascal Longlade, Trophos SA, Luminy Biotech Enterprises, Marseille, France; Jan Erik Nordrehaug, Institute of Medicine, University of Bergen, Bergen, Norway; Carole Perez, Trophos SA, Luminy Biotech Enterprises, Marseille, France; Rebecca Pruss, Trophos SA, Luminy Biotech Enterprises, Marseille, France; Gilles Sonou, Mobile Health, Paris, France; Sophie Schaller, Trophos SA, Luminy Biotech Enterprises, Marseille, France; Vegard Tuseth, Institute of Medicine, University of Bergen, Bergen, Norway; Eric Vicaut, Clinical Research Unit, Lariboisière Hospital, Paris, France.

\section{Acknowledgment}

The MITOCARE project is supported by the EU-FP7 for RTD - Project MITOCARE (Grant Agreement No. HEALTH-2010-261034). One of the beneficiaries of this grant is Trophos SA, Marseille, France.

In addition, clinical results will be compared to those limitations for use in research on the pathophysiology of reperfusion injury and for testing potential therapies.

References
1 Yellon DM, Hausenloy DJ: Myocardial reperfusion injury. N Engl J Med 2007;357: 1121-1135.

-2 Santoro GM, Carrabba N, Migliorini A, Parodi G, Valenti R: Acute heart failure in patients with acute myocardial infarction treated with primary percutaneous coronary intervention. Eur J Heart Fail 2008;10:780-785. 
-3 Staat P, Rioufol G, Piot C, Cottin Y, Cung TT, L'Huillier I, Aupetit JF, Bonnefoy E, Finet G, Andre-Fouet X, Ovize M: Postconditioning the human heart. Circulation 2005;112: 2143-2148.

-4 Piot C, Croisille P, Staat P, Thibault H, Rioufol G, Mewton N, Elbelghiti R, Cung TT, Bonnefoy E, Angoulvant D, Macia C, Raczka F, Sportouch C, Gahide G, Finet G, AndreFouet X, Revel D, Kirkorian G, Monassier JP, Derumeaux G, Ovize M: Effect of cyclosporine on reperfusion injury in acute myocardial infarction. N Engl J Med 2008;359: 473-481.

-5 Atar D, Petzelbauer P, Schwitter J, Huber K, Rensing B, Kasprzak JD, Butter C, Grip L, Hansen PR, Suselbeck T, Clemmensen PM, Marin-Galiano M, Geudelin B, Buser PT: Effect of intravenous FX06 as an adjunct to primary percutaneous coronary intervention for acute ST-segment elevation myocardial infarction results of the F.I.R.E. (Efficacy of FX06 in the Prevention of Myocardial Reperfusion Injury) trial. J Am Coll Cardiol 2009;53:720-729.

6 Thuny F, Lairez O, Roubille F, Mewton N, Rioufol G, Sportouch C, Sanchez I, Bergerot C, Thibault H, Cung TT, Finet G, Argaud L, Revel D, Derumeaux G, Bonnefoy-Cudraz E, Elbaz M, Piot C, Ovize M, Croisille P: Postconditioning reduces infarct size and edema in patients with ST-segment elevation myocardial infarction. J Am Coll Cardiol 2012; 59:2175-2181.

7 Thibault H, Piot C, Staat P, Bontemps L, Sportouch C, Rioufol G, Cung TT, Bonnefoy E, Angoulvant D, Aupetit JF, Finet G, AndreFouet X, Macia JC, Raczka F, Rossi R, Itti R, Kirkorian G, Derumeaux G, Ovize M: Longterm benefit of postconditioning. Circulation 2008;117:1037-1044.

8 Botker HE, Kharbanda R, Schmidt MR, Bottcher M, Kaltoft AK, Terkelsen CJ, Munk $\mathrm{K}$, Andersen NH, Hansen TM, Trautner S, Lassen JF, Christiansen EH, Krusell LR, Kristensen SD, Thuesen L, Nielsen SS, Rehling M, Sorensen HT, Redington AN, Nielsen TT: Remote ischaemic conditioning before hospital admission, as a complement to angioplasty, and effect on myocardial salvage in patients with acute myocardial infarction: a randomised trial. Lancet 2010;375:727-734.

-9 Yang XC, Liu Y, Wang LF, Cui L, Wang T, Ge YG, Wang HS, Li WM, Xu L, Ni ZH, Liu SH, Zhang L, Jia HM, Vinten-Johansen J, Zhao ZQ: Reduction in myocardial infarct size by postconditioning in patients after percutaneous coronary intervention. J Invasive Cardiol 2007;19:424-430.

10 Lonborg J, Kelbaek H, Vejlstrup N, Jorgensen E, Helqvist S, Saunamaki K, Clemmensen P, Holmvang L, Treiman M, Jensen JS, Engstrom T: Cardioprotective effects of ischemic postconditioning in patients treated with primary percutaneous coronary intervention, evaluated by magnetic resonance. Circ Cardiovasc Interv 2010;3:34-41.
Freixa X, Bellera N, Ortiz-Perez JT, Jimenez M, Pare C, Bosch X, De Caralt TM, Betriu A, Masotti M: Ischaemic postconditioning revisited: lack of effects on infarct size following primary percutaneous coronary intervention. Eur Heart J 2012;33:103-112.

12 Tarantini G, Favaretto E, Marra MP, Frigo AC, Napodano M, Cacciavillani L, Giovagnoni A, Renda P, De Biasio V, Plebani M, Mion M, Zaninotto M, Isabella G, Bilato C, Iliceto S: Postconditioning during coronary angioplasty in acute myocardial infarction: the POST-AMI trial. Int J Cardiol 2012, Epub ahead of print.

13 Bordet T, Buisson B, Michaud M, Drouot C, Galea P, Delaage P, Akentieva NP, Evers AS, Covey DF, Ostuni MA, Lacapere JJ, Massaad C, Schumacher M, Steidl EM, Maux D, Delaage M, Henderson CE, Pruss RM: Identification and characterization of cholest-4-en3-one, oxime (TRO19622), a novel drug candidate for amyotrophic lateral sclerosis. J Pharmacol Exp Ther 2007;322:709-720.

14 Schaller S, Paradis S, Ngoh GA, Assaly R, Buisson B, Drouot C, Ostuni MA, Lacapere JJ, Bassissi F, Bordet T, Berdeaux A, Jones SP, Morin D, Pruss RM: TRO40303, a new cardioprotective compound, inhibits mitochondrial permeability transition. J Pharmacol Exp Ther 2010;333:696-706.

15 Baines CP: The mitochondrial permeability transition pore and ischemia-reperfusion injury. Basic Res Cardiol 2009;104:181-188.

16 Javadov S, Karmazyn M, Escobales N: Mitochondrial permeability transition pore opening as a promising therapeutic target in cardiac diseases. J Pharmacol Exp Ther 2009;330:670-678.

- 17 Burns RJ, Gibbons RJ, Yi Q, Roberts RS, Miller TD, Schaer GL, Anderson JL, Yusuf S: The relationships of left ventricular ejection fraction, end-systolic volume index and infarct size to six-month mortality after hospital discharge following myocardial infarction treated by thrombolysis. J Am Coll Cardiol 2002;39:30-36.

18 Gibbons RJ, Miller TD, Christian TF: Infarct size measured by single photon emission computed tomographic imaging with $(99 \mathrm{~m})$ Tc-sestamibi: a measure of the efficacy of therapy in acute myocardial infarction. Circulation 2000;101:101-108.

19 Wright J, Bogaert J: Role of cardiac magnetic resonance imaging in ischaemic heart disease. Intern Med J 2009;39:563-573.

20 Pennell DJ, Sechtem UP, Higgins CB, Manning WJ, Pohost GM, Rademakers FE, van Rossum AC, Shaw LJ, Yucel EK: Clinical indications for cardiovascular magnetic resonance (CMR): Consensus Panel report. Eur Heart J 2004;25:1940-1965.
21 Kim RJ, Fieno DS, Parrish TB, Harris K, Chen EL, Simonetti O, Bundy J, Finn JP, Klocke FJ, Judd RM: Relationship of MRI delayed contrast enhancement to irreversible injury, infarct age, and contractile function. Circulation 1999;100:1992-2002.

22 Hedstrom E, Engblom H, Frogner F, Astrom-Olsson $\mathrm{K}$, Ohlin $\mathrm{H}$, Jovinge S, Arheden $\mathrm{H}$ : Infarct evolution in man studied in patients with first-time coronary occlusion in comparison to different species - implications for assessment of myocardial salvage. J Cardiovasc Magn Reson 2009;11:38.

23 Aletras AH, Tilak GS, Natanzon A, Hsu LY, Gonzalez FM, Hoyt RF Jr, Arai AE: Retrospective determination of the area at risk for reperfused acute myocardial infarction with T2-weighted cardiac magnetic resonance imaging: histopathological and displacement encoding with stimulated echoes (DENSE) functional validations. Circulation 2006;113:1865-1870.

24 Carlsson M, Ubachs JF, Hedstrom E, Heiberg E, Jovinge S, Arheden H: Myocardium at risk after acute infarction in humans on cardiac magnetic resonance: quantitative assessment during follow-up and validation with single-photon emission computed tomography. JACC Cardiovasc Imaging 2009; 2:569-576.

25 Sorensson P, Heiberg E, Saleh N, Bouvier F, Caidahl K, Tornvall P, Ryden L, Pernow J, Arheden H: Assessment of myocardium at risk with contrast enhanced steady-state free precession cine cardiovascular magnetic resonance compared to single-photon emission computed tomography. J Cardiovasc Magn Reson 2010;12:25.

26 Ubachs JF, Sorensson P, Engblom H, Carlsson $\mathrm{M}$, Jovinge $\mathrm{S}$, Pernow J, Arheden $\mathrm{H}$ : Myocardium at risk by magnetic resonance imaging: head-to-head comparison of T2weighted imaging and contrast-enhanced steady-state free precession. Eur Heart J Cardiovasc Imaging 2012, DOI: 10.1093/ehjci/ jes1091.

27 Heiberg E, Ugander M, Engblom H, Gotberg M, Olivecrona GK, Erlinge D, Arheden H: Automated quantification of myocardial infarction from MR images by accounting for partial volume effects: animal, phantom, and human study. Radiology 2008;246:581588.

-28 Engblom H, Hedstrom E, Heiberg E, Wagner GS, Pahlm O, Arheden H: Rapid initial reduction of hyperenhanced myocardium after reperfused first myocardial infarction suggests recovery of the peri-infarction zone: one-year follow-up by MRI. Circ Cardiovasc Imaging 2009;2:47-55.

-29 Ibrahim T, Hackl T, Nekolla SG, Breuer M, Feldmair M, Schomig A, Schwaiger M: Acute myocardial infarction: serial cardiac MR imaging shows a decrease in delayed enhancement of the myocardium during the 1st week after reperfusion. Radiology 2010;254:8897. 